Article

\title{
Probabilistic Representation Approach for Multiple Types of Epistemic Uncertainties Based on Cubic Normal Transformation
}

\author{
Xiang Peng ${ }^{1,2} \mathbb{D}$, Qilong Gao ${ }^{1}$, Jiquan $\mathrm{Li}^{1}$, Zhenyu Liu ${ }^{2}$, Bing $\mathrm{Yi}^{3}$ and Shaofei Jiang ${ }^{1, *}$ \\ 1 College of Mechanical Engineering, Zhejiang University of Technology, Hangzhou 310023, China; \\ pengxiang@zjut.edu.cn (X.P.); gao_qilong@163.com (Q.G.); lijq@zjut.edu.cn (J.L.) \\ 2 State Key Laboratory of Fluid Power and Mechatronic Systems, Zhejiang University, Hangzhou 310027, \\ China; liuzy@zju.edu.cn \\ 3 School of Traffic and Transportation Engineering, Central South University, Changsha 410075, China; \\ bingyi@csu.edu.cn \\ * Correspondence: jsf75@zjut.edu.cn; Tel./Fax: +86-571-8529-0368
}

Received: 5 June 2020; Accepted: 6 July 2020; Published: 8 July 2020

\begin{abstract}
Many non-probabilistic approaches have been widely regarded as mathematical tools for the representation of epistemic uncertainties. However, their heavy computational burden and low computational efficiency hinder their applications in practical engineering problems. In this article, a unified probabilistic representation approach for multiple types of epistemic uncertainties is proposed based on the cubic normal transformation method. The epistemic uncertainties can be represented using an interval approach, triangular fuzzy approach, or evidence theory. The uncertain intervals of four statistical moments, which contain mean, variance, skewness, and kurtosis, are calculated using the sampling analysis method. Subsequently, the probabilistic cubic normal distribution functions are conducted for sampling points of four statistical moments of epistemic uncertainties. Finally, a calculation procedure for the construction of probabilistic representation functions is proposed, and these epistemic uncertainties are represented with belief and plausibility continuous probabilistic measure functions. Two numerical examples and one engineering example demonstrate that the proposed approach can act as an accurate probabilistic representation function with high computational efficiency.
\end{abstract}

Keywords: uncertainty analysis; epistemic uncertainty; cubic normal transformation; statistical moments

\section{Introduction}

There are various sources of uncertainties in real-world engineering conditions, such as loading uncertainties, material uncertainties, geometric and boundary uncertainties due to manufacturing tolerances, variation in operating environments, and differences in technical levels. A small amount of uncertainties may have a severe influence on the overall performance of a system owing to the nonlinear coupling uncertainty propagation of multiple uncertain variables in the complex engineering problems. Therefore, uncertainties should be appropriately quantified and analyzed to improve the performance of engineering products in actual situations.

The uncertainties are assumed to be aleatory uncertainties, which have determinate and complete uncertain information, and are represented using accurate probability density functions (PDFs). Many probabilistic uncertain analysis and optimization methodologies have been proposed to decrease the deteriorative effects of uncertainties and improve the reliability of engineering products, such as the first-order reliability design method [1-3], the second-order reliability design method [4-6], the time-dependent reliability design method [7], and the reliability-based robust design method [8]. 
However, it is difficult to acquire an accurate PDF of uncertainties in some actual engineering applications. Only insufficient information can be acquired, and the economic cost or computational complexity is too high to acquire further uncertainty information. New uncertainty problems are produced when using aleatory uncertainties to address these uncertainty problems, the reliability design becomes the calculation of the uncertainty of uncertain variables, and the computational complexity increases exponentially with the number of uncertain variables, which makes it impossible to calculate in complex engineering problems.

To address the problems of incomplete information or insufficient data for uncertain variables, the use of epistemic uncertainty is proposed. This has been widely applied to the uncertainty analysis and reliability design of engineering products [9-11]. Some non-probabilistic uncertainty analysis methods have been proposed to address epistemic uncertainties, and summarized by Beer [12], which includes the interval analysis approach $[13,14]$, the convex model $[15,16]$, the fuzzy set $[17,18]$, and the evidence theory [19-22]. These non-probabilistic analysis methods have different application situations based on the available uncertainty information. The interval analysis method is used when the uncertainty information is lacking, only lower and upper bounds of uncertain variables can be acquired. The convex model discerns the boundaries of uncertain variables instead of their precise probability distributions, which can be represented with a few sample information. The interval model and ellipsoid convex model are two common convex models [23]. When some quantitative uncertainty information is available, fuzzy set theory can be used, and the uncertainty is represented using membership functions. Evidence theory uses belief assignment as its basis to describe uncertainty information, and then it represents the variable uncertainty with the belief and plausibility measure function. With these non-probabilistic uncertainty representation methods, epistemic uncertainty is quantified, propagated, and optimized in the reliability design of engineering products [24,25].

However, there are some challenges in the application of these non-probabilistic uncertainty representation methods for actual engineering problems. One of the challenges is the high computational cost [26], which is caused by the discretized quantification of epistemic uncertainties. The calculation complexity increases with the number of epistemic variables and the discrete interval numbers of all epistemic variables. Another challenge is the unified calculation of aleatory uncertainty and epistemic uncertainty. The reliability design problem with aleatory and epistemic uncertainties is simultaneously a multiple layer nesting optimization problem. Common gradient optimization methods cannot be implemented because of the discontinuity of epistemic uncertainties.

To improve the computational efficiency and reduce the complexity of reliability design, some probabilistic representation methods for epistemic uncertainties have been proposed, and the unified uncertainty calculations of aleatory and epistemic uncertainties have been implemented [27-29]. Sankararaman [30-32] presented a likelihood-based probabilistic representation method of epistemic uncertainty for which only sparse point and/or interval data are available, and quantified the individual contributions of variability and uncertain distribution parameters using Bayesian model averaging and Bayesian hypothesis testing approaches. Zaman [33-35] proposed a probabilistic framework for the representation and propagation of uncertain interval variables, which were fitted to the Johnson distribution using the moment matching approach. Then, the proposed framework was applied to the robust optimization design of engineering problems. Cho [36] presented the Bayesian approach and Monte Carlo method to take insufficient input data into consideration in reliability optimization design, and then determined the probability of failure probability and reliability of optimum results. Lü [37] proposed equivalent evidence-based fuzzy variables to represent the interval variables, fuzzy variables, and evidence theory variables simultaneously. Zhang [38] employed the multi-model inference method to quantify and propagate the uncertainty created by a lack of sufficient statistical data, and applied the proposed algorithm into uncertainty analysis of the plate buckling strength. Zhao [39,40] derived a complete probabilistic expression of epistemic uncertainties according to the mean, variance, skewness, and kurtosis of available statistical data, and applied the proposed cubic normal distribution in a variety of areas in reliability engineering. Zhang [41] and Liu [42] constructed Johnson p-boxes to 
equivalently represent evidence variables, and converted the uncertainty analysis problem of evidence theory into two rounds of probabilistic uncertainty analysis problem.

Though a series of research studies about the probabilistic representation of epistemic uncertainties have been published, there are still some limitations in this area:

(1) In the actual engineering product, there are multiple types of epistemic uncertainties with different representation functions according to the available uncertainty information. Multiple different epistemic uncertainties cannot be represented in a unified probabilistic analysis framework. Lü [37] used the evidence-based fuzzy variable to represent multiple types of epistemic uncertainties, but the variables were calculated using the non-probabilistic uncertain analysis method.

(2) The representation function of epistemic uncertainties is discontinuous, so repeated extreme analysis of epistemic uncertainties at discrete subintervals should be implemented, which reduces the computational efficiency in the reliability design of engineering products.

To resolve these issues effectively, a unified probabilistic uncertainty representation approach for multiple types of epistemic uncertainties is proposed based on the cubic normal distribution method. The cubic normal distribution method has been applied to the probabilistic uncertainty representation of interval variables [43] and probabilistic variables with unknown distribution types [44], which is effective in the calculation of the reliability index and reliability design $[45,46]$. Therefore, this article extends the application range of the cubic normal transformation method from the representation of interval variables to probabilistic representation for multiple types of epistemic uncertainties. The calculation algorithm of four statistical moments for triangle fuzzy and evidence theory variables are proposed, and the unified probabilistic representation framework is proposed for uncertainty propagation analysis. The remainder of this article is organized as follows. Three non-probabilistic representation methods for epistemic uncertainties are introduced in Section 2: The interval approach, triangular fuzzy approach, and evidence theory. The calculation methods of four statistical moments, which includes the mean, variance, skewness, and kurtosis, are proposed for the three types of epistemic uncertainties in Section 3. In Section 4, the unified cubic normal distribution function is calculated using the moments matching method, and the corresponding continuous probability distribution functions are constructed for epistemic uncertainties. Two mathematical examples and one engineering example are illustrated in Section 5. The study's conclusions and recommendations for future research are presented in Section 6.

\section{Multiple Types of Non-Probabilistic Representation Approaches for Epistemic Uncertainties}

\subsection{Interval Approach}

Two interval representation approaches for epistemic uncertainty are considered: (1) Single interval, and (2) multiple intervals. In some situations, the sampling information of epistemic uncertainty cannot be obtained, and only possible boundaries of epistemic uncertainty are acquired through theoretical calculation or physical analysis, the single interval is used to represent epistemic uncertainty. In other situations, the uncertain information can be determined using experiments or measurements, but the observation data are intervals because of limitations of the measuring equipment. Therefore, multiple interval information is available for the representation of epistemic uncertainty.

In the single interval approach, the epistemic uncertainty $x$ is represented using an interval vector:

$$
x \in[\underline{x}, \bar{x}], \underline{x}=x^{c}-\Delta x, \bar{x}=x^{c}+\Delta x,
$$

where $\underline{x}, \bar{x}, x^{c}$, and $\Delta x$ are the lower bound, upper bound, midpoint, and radius of epistemic uncertainty, respectively.

For an engineering problem whose uncertain input variables $\mathbf{x}$ are represented using the single interval method, the system response function $y=f(\mathbf{x})$ is also an interval variable. The uncertainty of $y$ can be calculated using a series of available methods, such as the perturbation analysis method and reliability index method. 
In the multiple interval approach, the epistemic uncertainty $x$ is represented using $m$ intervals $\left[a_{i}, b_{i}\right], i=1, \cdots, m$, where $a_{i}$ and $b_{i}$ are the lower and upper bounds of the $i$-th subinterval, respectively. These subintervals can be overlapping or non-overlapping intervals. These multiple intervals include partial uncertainty information of $x$. In actual engineering problems, many uncertain variables $\mathbf{x}$ represented using the multiple interval approach should be included, and then the uncertainty of the response function $y=f(\mathbf{x})$ can be analyzed.

\subsection{Triangular Fuzzy Approach}

Fuzzy set theory was introduced by Zadeh [47] and has been widely applied to various fields, such as uncertainty analysis, information fusion, decision-making, and clustering. A fuzzy set $\Psi$ is defined as a pair $\Psi=(x, \mu(x))$, where $x \in \Omega$ is an element in the domain $\Omega$, and $\mu(x)$ is the membership function that can be defined as $\mu(x) \in[0,1]$. The $\alpha$ level of the fuzzy set $\Psi$ is defined as $\Psi^{\alpha}=\{x \in \Omega \mid \mu(x)=\alpha\}$, and the $\alpha$-cut of the fuzzy set is defined as $\Lambda_{A}^{\alpha}=\{x \in \Omega \mid \mu(x) \geq \alpha, \alpha \in[0,1]\}$. For each $\alpha$-cut level, the uncertain interval of $x$ is obtained as $\left[x_{\alpha}^{L}, x_{\alpha}^{U}\right] . x_{\alpha}^{L}$ and $x_{\alpha}^{U}$ are the lower and upper bound of $x$, which are calculated based on the value of $\alpha$ and the membership function of $\mu(x)$.

The shape of $\mu(x)$ may be arbitrary, and many different calculation methods for the fuzzy set have been proposed, such as the L-fuzzy set, triangular fuzzy number, and fuzzy rough sets. The triangular fuzzy number is the most common representation model of the fuzzy set. The membership function $\mu(x)$ is defined in Equation (2), where $b$ is the most likely value, and $a$ and $c$ are the lower and upper bound of $x$, respectively. For convenience, the triangular fuzzy number can be denoted by $[a, b, c]$. The lower bound $x_{\alpha}^{L}$ and upper bound $x_{\alpha}^{U}$ by $\alpha$ cut of the membership function are shown in Figure 1:

$$
\mu(x)=\left\{\begin{array}{cc}
0 & x<a \\
\frac{x-a}{b-a} & a \leq x \leq b \\
\frac{c-x}{c-b} & b \leq x \leq c \\
0 & x>c
\end{array}\right.
$$

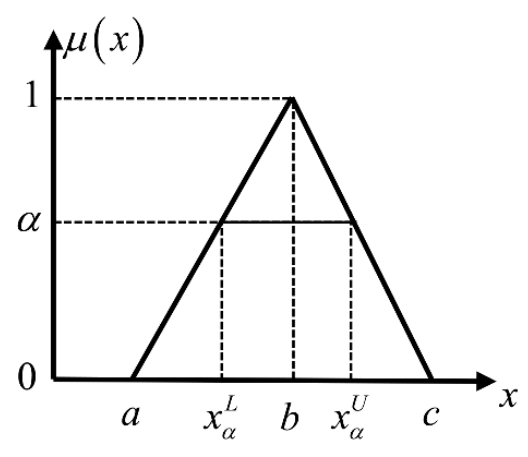

Figure 1. Triangular fuzzy number and its $\alpha$-cut.

\subsection{Evidence Theory}

Evidence theory, which is also called the Dempster-Shafer theory, defines a framework of discernment for epistemic uncertainty $x$, which consists of all possible elementary propositions. The power set of all possible propositions $\Omega$ for $x$ is composed of $m x$ subintervals $\Theta_{i}(i=1, \cdots, m x)$. For every subinterval $\Theta_{i} \in \Omega$, the basic probability assignment (BPA) $m\left(\Theta_{i}\right)$ satisfies the three axioms given in Equations (3)-(5):

$$
\begin{gathered}
m\left(\Theta_{i}\right) \geq 0, \forall \Theta_{i} \subseteq \Omega, \\
m(\varnothing) \geq 0,
\end{gathered}
$$




$$
\sum_{i=1}^{m x} m\left(\Theta_{i}\right)=1 .
$$

For the response function $y=f(\mathbf{x})$, where $\mathbf{x}$ is multiple epistemic uncertainties represented using evidence theory, and the failure probability of $y$ is characterized using belief and plausibility measures. The belief measure $\operatorname{Bel}(Y)$ of the failure event $\ell \in Y$ is the sum of the BPA of subsets of evidence theory variables $x$ entirely within the failure region $Y=\{f(\mathbf{x}) \leq \psi\}$, and the plausibility measure $\operatorname{Pl}(Y)$ of the failure event $\ell \in Y$ is calculated by adding the BPAs of the subsets of evidence theory variables $\mathbf{x}$, which are in the failure region and intersect with the failure region:

$$
\begin{aligned}
& \operatorname{Bel}(Y)=\sum_{\psi \subseteq Y} m(\psi), \\
& \operatorname{Pl}(Y)=\sum_{\psi \cap Y \neq \varnothing} m(\psi) .
\end{aligned}
$$

The uncertainty of the response function $y$ is represented using interval $[\operatorname{Bel}(Y), \operatorname{Pl}(Y)]$.

\section{Unified Calculation of Four Statistical Moments of Epistemic Uncertainties}

\subsection{Four Statistical Moments}

By using similar methods for discrete random variables with determinate PDF, the first-order moment (mean) $M_{1}$, second-order moment (variance) $M_{2}$, third-order moment (skewness) $M_{3}$, and the fourth-order moment (kurtosis) $M_{4}$ of epistemic uncertainty $x$ can be calculated through Equations (8)-(11):

$$
\begin{gathered}
M_{1}=E(x), \\
M_{2}=E\left(x^{2}\right)-(E(x))^{2}, \\
M_{3}=E\left(x^{3}\right)-3 E\left(x^{2}\right) E(x)+2(E(x))^{3}, \\
M_{4}=E\left(x^{4}\right)-4 E\left(x^{3}\right) E(x)+6 E\left(x^{2}\right)(E(x))^{2}-3(E(x))^{4},
\end{gathered}
$$

where $E(x)=\sum_{i=1}^{n s} \widetilde{x}_{i} p\left(\widetilde{x}_{i}\right), E\left(x^{2}\right)=\sum_{i=1}^{n s} \widetilde{x}_{i}^{2} p\left(\widetilde{x}_{i}\right), E\left(x^{3}\right)=\sum_{i=1}^{n s} \widetilde{x}_{i}^{3} p\left(\widetilde{x}_{i}\right), E\left(x^{4}\right)=\sum_{i=1}^{n s} \widetilde{x}_{i}^{4} p\left(\widetilde{x}_{i}\right), \widetilde{x}_{i}$, and $p\left(\widetilde{x}_{i}\right)$ are the random sampling points and corresponding probability density values, and $n s$ is the number of sampling points.

For epistemic uncertainty $x$, it is impossible to obtain an accurate PDF of $x$, as the values of $E\left(x^{l}\right)$ change with the sampling points and their probability density values. Therefore, the calculated four statistical moments $M_{1} \sim M_{4}$ are variational and are expressed as intervals $\left[M_{l}^{L}, M_{l}^{U}\right], l=1,2,3,4$. The bounds of $M_{1} \sim M_{4}$ are estimated using the optimization methods presented in [48]:

$$
M_{l}^{L} / M_{l}^{U}=\underset{p\left(\widetilde{x}_{i}\right)}{\min } \max M_{l}
$$

where $n s$ sampling points $\widetilde{x}_{i}(i=1, \cdots, n s)$ are determinate, and are uniformly distributed in the uncertain design space of epistemic uncertainty. The probability density values of these sampling points $p\left(\widetilde{x}_{i}\right)$ are optimization variables that are constrained by the specific uncertainty types of epistemic uncertainty. For example, if the epistemic uncertainty $x$ is represented using a single interval, the probability density values $p\left(\widetilde{x}_{i}\right)$ are random values in $[0,1]$, and the total probability density values of $n s$ sampling points $\widetilde{x}_{i}(i=1, \cdots, n s)$ are equal to 1 . The optimization formulations presented in Equation (12) are solved using the MATLAB function fmincon, which is implemented using the sequential quadratic programming algorithm with random initial points. The optimization algorithm is repeatedly implemented several times to reduce the random error due to the initial points. If the 
epistemic uncertainty is represented using the interval approach, regardless of whether the available uncertainty information is a single interval or multiple intervals, the four statistical moments are calculated using the method of Zaman [35] based on the theory presented in Equations (8)-(12)

\subsection{Moments Calculation for Triangular Fuzzy Variable}

If the epistemic uncertainty $x$ is represented using the triangular fuzzy approach, the lower bound, likely value, and upper bound are $a, b$, and $c$, respectively. The $l$-order moments under the $\alpha$ level are calculated using Equation (13) [49]:

$$
E\left(x^{l}\right)=(1-\alpha) \frac{b^{l+1}-a^{l+1}}{(l+1)(b-a)}+\alpha \frac{b^{l+1}-c^{l+1}}{(l+1)(b-c)}, l=1, \cdots, 4 .
$$

After determining the 1 4 order moments of the triangular fuzzy variable using Equation (13), the four statistical moments $M_{1} \sim M_{4}$ for triangular fuzzy variable are calculated using Equations (8)-(11). Then, the lower and upper bounds of $M_{1} \sim M_{4}$ for a triangular fuzzy variable are determined using Equation (12). The four moments for the triangular fuzzy variable $A=[1,1.3,2]$ are shown in Figure 2 . Results indicate that the first-order moment $M_{1}$ is a linear function of $\alpha$, while the second-order moment $M_{2}$, third-order moment $M_{3}$, and fourth-order moment $M_{4}$ are nonlinear functions of $\alpha$. The maximum values of $M_{1} \sim M_{4}$ are determined at different $\alpha$ values. Using the optimization method in Equation (12), the uncertainty intervals of $M_{1} \sim M_{4}$ are [1.15, 1.65], [0.008, 0.088], [-0.005, 0.021], and $[0,0.015]$, respectively.

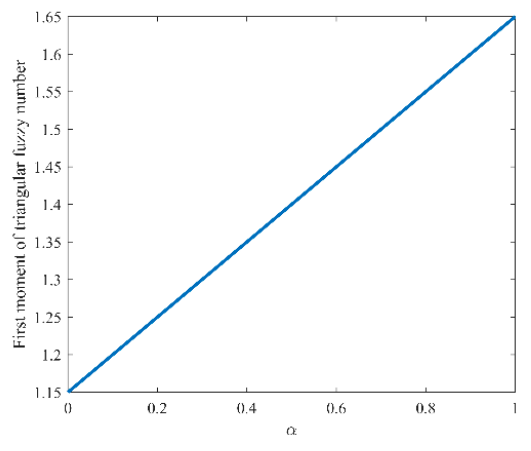

(a)

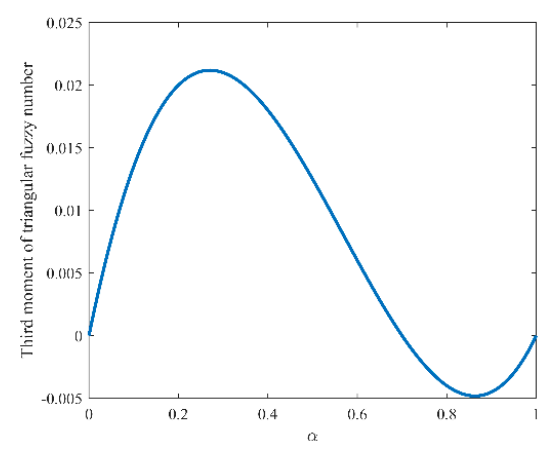

(c)

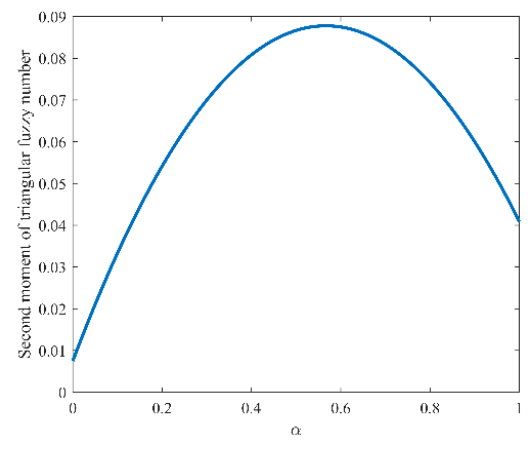

(b)

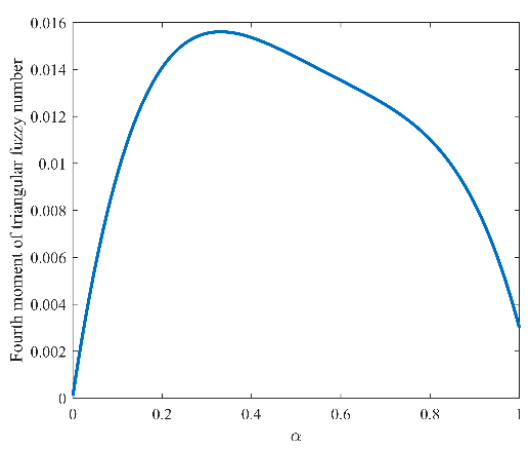

(d)

Figure 2. Four moments of the triangular fuzzy variables (a) The first-order moment (mean); (b) Secondorder moment (variance); (c) Third-order moment (skewness); (d) Fourth-order moment (kurtosis).

\subsection{Moments Calculation for Evidence Theory Variable}

The epistemic uncertainty, represented using evidence theory, can be regarded as a summation of multiple uncertain single intervals whose weight ratios are their corresponding BPAs. The $l$ th-order 
origin mean $E\left(x^{l}\right), l=1,2,3,4$ of epistemic uncertainty $x$ is a function of BPAs and the mean of every subinterval.

If the epistemic uncertainty $x$ is represented using two subintervals $x_{1}=\left[\underline{x}_{1}, \bar{x}_{1}\right]$ and $x_{2}=\left[\underline{x}_{2}, \bar{x}_{2}\right]$ with corresponding BPAs $w_{1}$ and $w_{2}$, respectively, $E\left(x^{l}\right)$ is calculated using Equations (14)-(17):

$$
\begin{gathered}
E(x)=w_{1} E\left(x_{1}\right)+w_{2} E\left(x_{2}\right), \\
E\left(x^{2}\right)=w_{1}^{2} E\left(x_{1}^{2}\right)+2 w_{1} w_{2} E\left(x_{1}\right) E\left(x_{2}\right)+w_{2}^{2} E\left(x_{2}^{2}\right), \\
E\left(x^{3}\right)=w_{1}^{3} E\left(x_{1}^{3}\right)+3 w_{1}^{2} w_{2} E\left(x_{1}^{2}\right) E\left(x_{2}\right)+3 w_{1} w_{2}^{2} E\left(x_{1}\right) E\left(x_{2}^{2}\right)+w_{2}^{3} E\left(x_{2}^{3}\right), \\
E\left(x^{4}\right)=w_{1}^{4} E\left(x_{1}^{4}\right)+4 w_{1}^{3} w_{2} E\left(x_{1}^{3}\right) E\left(x_{2}\right)+6 w_{1}^{2} w_{2}^{2} E\left(x_{1}^{2}\right) E\left(x_{2}^{2}\right) \\
+4 w_{1} w_{2}^{3} E\left(x_{1}\right) E\left(x_{2}^{3}\right)+w_{2}^{4} E\left(x_{2}^{4}\right)
\end{gathered}
$$

where $w_{1}+w_{2}=1, E\left(x_{1}^{l}\right)$ and $E\left(x_{2}^{l}\right)$ are the l-order means of subintervals $x_{1}$ and $x_{2}$, respectively. It is impossible to acquire an accurate PDF in every subinterval. $E\left(x_{1}^{l}\right)$ and $E\left(x_{2}^{l}\right)$ are calculated to be intervals. Therefore, the four statistical moments $M_{1} \sim M_{4}$ are also intervals, and the lower and upper bounds of $M_{1} \sim M_{4}$ are calculated using the optimization method presented in Equation (12). An evidence theory variable is shown as an example, which is composed of three subintervals $[10,13]$, $[13,17]$, and $[17,20]$, with BPAs of $0.2,0.7$, and 0.1 , respectively. The histograms of $M_{1} \sim M_{4}$ are shown in Figure 3. Using the optimization method presented in Equation (12), the uncertain intervals of $M_{1} \sim M_{4}$ are shown to be $[13.87,15.48],[3.51,6.66],[-10.01,9.46]$, and $[34.25,103.51]$, respectively.

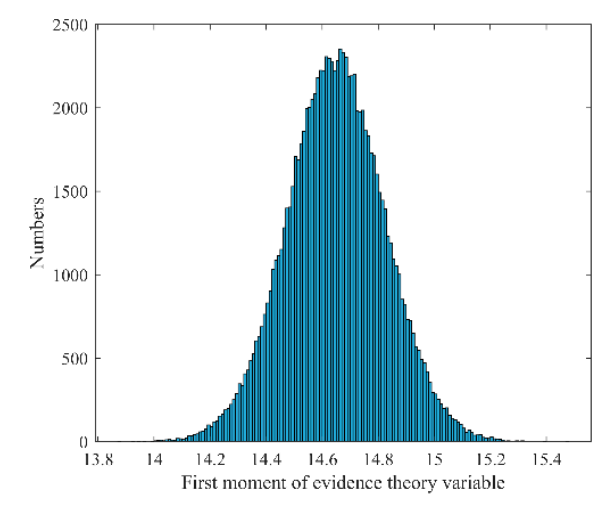

(a)

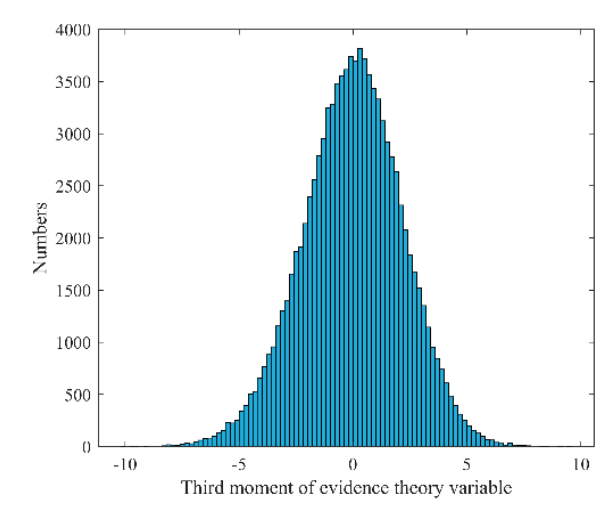

(c)



(b)

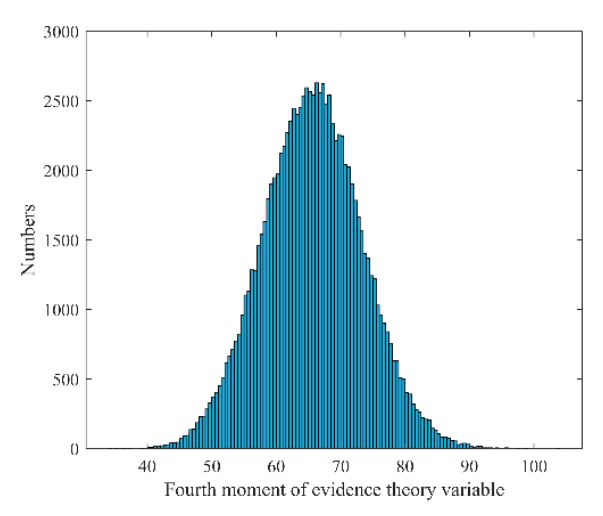

(d)

Figure 3. Four moments of the evidence theory variable (a) First-order moment (mean); (b) second-order moment (variance); (c) third-order moment (skewness); (d) fourth-order moment (kurtosis). 


\section{Fitting Cubic Normal Distribution of Epistemic Uncertainties}

After determining the uncertain intervals of four statistical moments of epistemic uncertainties represented using the interval approach, triangular fuzzy approach, or evidence theory, the cubic normal distribution functions are fitted to probabilistically describe these epistemic uncertainties simultaneously.

\subsection{Cubic Normal Distribution}

After determining the first four statistical moments $M_{1} \sim M_{4}$ of epistemic uncertainties $x$, the standardized variable $x_{s}$ of $x$ is transferred to a cubic function of a random variable $u$ using Equation (18):

$$
\frac{x-\mu_{x}}{\sigma_{x}}=x_{s}=S_{u}(u)=a_{4} u^{3}+a_{3} u^{2}+a_{2} u+a_{1},
$$

where $\mu_{x}=M_{1}$ and $\sigma_{x}=\sqrt{M_{2}}$ are the mean and standard deviation of $x$, respectively. $a_{1} \sim a_{4}$ are the functions of four statistical moments $M_{1} \sim M_{4}$; the calculation details can be found in [39].

After determining coefficients $a_{1} \sim a_{4}$, the complete monotonic expression of $u$ is decomposed into six types according to the values of $q=\frac{3 a_{2} a_{4}-a_{3}^{2}}{3 a_{4}^{2}}, a_{4}$, and $M_{3}$, which are summarized in Table 1 . The six types include unbounded distributions (Types I and VI), unilaterally bounded distributions (Types II, III, and V), and a bilaterally bounded distribution (Type IV). The definitions of parameters $A$, $B, \Theta, r, \theta, J_{1}^{*}$, and $J_{2}^{*}$ are illustrated in $[39,40]$.

Table 1. Complete monotonic expression of $u$.

\begin{tabular}{|c|c|c|c|c|c|}
\hline & \multicolumn{2}{|c|}{ Parameters } & $u$ & Range of $x$ & Type \\
\hline \multirow{3}{*}{$q \geq 0$} & \multirow[b]{3}{*}{$a_{4}>0$} & & $\sqrt[3]{A}+\sqrt[3]{B}-\Theta / 3$ & $(-\infty,+\infty)$ & IV \\
\hline & & $M_{3} \geq 0$ & $2 r \cos (\theta / 3)-\Theta / 3$ & $J_{1}^{*}<x<J_{2}^{*}$ & II \\
\hline & & & $\sqrt[3]{A}+\sqrt[3]{B}-\Theta / 3$ & $x \geq J_{2}^{*}$ & \\
\hline \multirow[t]{4}{*}{$q<0$} & & $M_{3}<0$ & $\begin{array}{c}\sqrt[3]{A}+\sqrt[3]{B}-\Theta / 3 \\
-2 r \cos [(\theta-\pi) / 3]-\Theta / 3\end{array}$ & $\begin{array}{c}x \leq J_{1}^{*} \\
I_{1}^{*}<x<I_{0}^{*}\end{array}$ & III \\
\hline & $a_{4}<0$ & & $-2 r \cos [(\theta+\pi) / 3]-\Theta / 3$ & $J_{2}^{*} \leq x \leq J_{1}^{*}$ & $\mathrm{I}$ \\
\hline & $a_{4}=0$ & $M_{3} \neq 0$ & $-a_{2}+\sqrt{a_{2}^{2}+4 a_{3}\left(a_{3}+x_{s}\right)}$ & $a_{2}^{2}+4 a_{3}\left(a_{3}+x_{s}\right) \geq 0$ & $\mathrm{~V}$ \\
\hline & & $M_{3}=0$ & $\begin{array}{l}x_{s} \\
x_{3}\end{array}$ & $(-\infty,+\infty)$ & VI \\
\hline
\end{tabular}

The PDF $p(x)$ of epistemic uncertainty $x$ is calculated based on the PDF $\phi(u)$ of $u$ using Equation (19):

$$
p(x)=\frac{\phi(u)}{\sigma_{x}\left(3 a_{4} u^{2}+2 a_{3} u+a_{2}\right)} .
$$

When the four statistical moments of epistemic uncertainty $x$ are determined, the transformational continuous PDF can be calculated using the above methods. The calculated PDF and cumulative density function $(\mathrm{CDF})$ of the standardized variable $x_{s}$ (mean $M_{1}=0$ and variance $M_{2}=1$ ) with different levels of skewness $M_{3}$ and kurtosis $M_{4}$ are shown in Figure 4. 


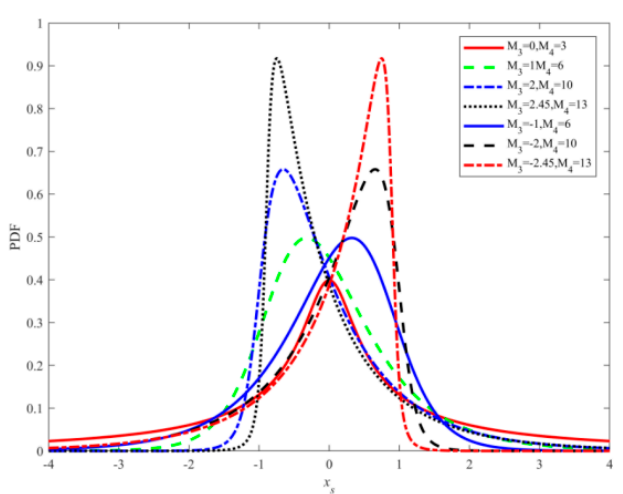

(a)

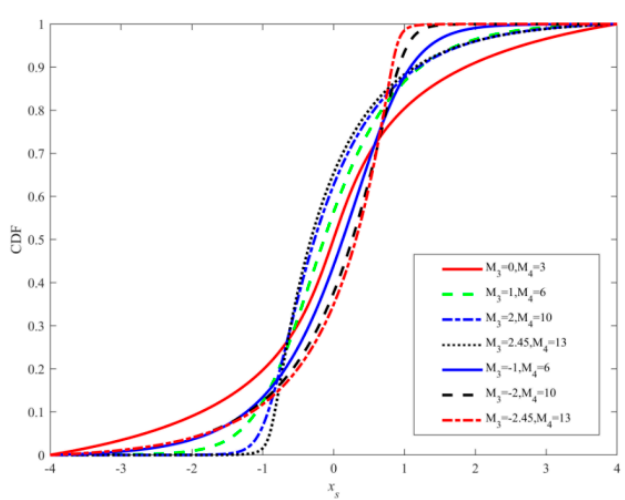

(b)

Figure 4. Representation functions of epistemic variables (a) probability density function (PDF) (b) cumulative CDF.

\subsection{Fit Continuous Probability Density Function}

Several approaches of constructing continuous Johnson distribution functions have been proposed to fit interval variables according to four statistical moments or percentiles. However, additional parameters $\gamma$ and $\delta$ must be optimized in the construction of every Johnson distribution under every sampling point of four statistical moments, which increases the calculation complexity. Therefore, a new probabilistic approach for simultaneous representation of interval variables, triangular fuzzy variables, and evidence theory variables is developed using cubic normal distributions, which do not require the calculation of additional parameters. The detailed procedure is shown as follows.

Step 1: The uncertain design interval of epistemic uncertainty $x$ is determined according to its non-probabilistic characterization method.

(1) $x$ is represented using the interval approach. If the available information is single interval $[\underline{x}, \bar{x}]$, the uncertain design interval $\left[x_{\text {lower }}, x_{\text {upper }}\right]$ of $x$ is also a single interval $[\underline{x}, \bar{x}]$. While the available information is presented in multiple intervals $\left[a_{i}, b_{i}\right], i=1, \cdots, m$, the lower bound $x_{\text {lower }}=\min \left\{a_{1}, \cdots, a_{m}\right\}$ is the minimum value of multiple lower bounds of the available intervals, and the upper bound $x_{\text {upper }}=\max \left\{b_{1}, \cdots, b_{m}\right\}$ is the maximum value of multiple upper bounds of the available intervals.

(2) $x$ is represented using the triangular fuzzy approach. The lower bound $x_{\alpha=0}^{L}$ and upper bound $x_{\alpha=0}^{U}$ at $\alpha=0$ construct the uncertain design interval of $x$.

(3) $x$ is represented using evidence theory. The lower bound $x_{\text {lower }}$ is the minimum value of the lower bounds of multiple subintervals, and the upper bound $x_{\text {upper }}$ is the maximum value of the upper bounds of multiple subintervals.

Step 2: The sampling points of epistemic uncertainty $x$ are available randomly in its uncertain design interval $\left[x_{\text {lower }}, x_{\text {upper }}\right]$ according to its uncertainty type.

(1) $x$ is represented by using the interval approach. If $x$ is represented with a single interval, random sampling points are available in the single interval. While $x$ is represented with multiple intervals $\left[a_{i}, b_{i}\right](i=1, \cdots, m)$, the same numbers of sampling points are obtained using the uniform sampling method in every subinterval $\left[a_{i}, b_{i}\right]$.

(2) $x$ is represented by using the triangular fuzzy approach. For the given $\alpha$ value, the uncertain design interval is decomposed into two subintervals $\left[x_{\alpha}^{L}, b\right]$ and $\left[b, x_{\alpha}^{U}\right]$, and the same number of sampling points are collected randomly in the two uncertainty subintervals.

(3) $x$ is represented by using evidence theory. The same number of sampling points is obtained in every subinterval, and then the total probability of every subinterval is set to be its basic probability assignment.

Step 3: The four statistical moments $M_{1} \sim M_{4}$ are calculated based on the sampling points in Step 2 using the methods in Section 3 for all types of epistemic uncertainties. 
Step 4: The continuous cubic normal distribution variable $u$ is calculated using the transformation method presented in Section 4.1. For every sampling value of $M_{1} \sim M_{4}$, the standardized variable $x_{s}$ for $x$ is calculated to be $x_{s}=\left(x-M_{1}\right) / \sqrt{M_{2}}$; the values of $p, a_{4}$, and $M_{3}$ are calculated; and then the uncertainty type of $u$ is chosen from type I to VI. The PDF of $u$ is calculated, and the PDF of $x$ is calculated using Equation (19).

Step 5: Steps 2-4 are repeated to construct a series of the cubic normal distribution function under different values of $M_{1} \sim M_{4}$, and then a series of PDF and the corresponding CDF of $x$ are gained.

Step 6: The uncertain design interval of $x$ is uniformly decomposed into 101 points $x^{\tau}=x_{\text {lower }}+0.01 *(\tau-1) *\left(x_{\text {upper }}-x_{\text {lower }}\right), \tau=1, \cdots, 101$, and the lower bound and upper bound of CDF values at these sampling points are calculated based on the CDF results in Step 5.

Step 7: The upper bound (Pl measure) of the continuous PDF of epistemic uncertainty $x$ is the curve of the upper bound at different points $x^{\tau}$, and the lower bound (Bel measure) of the continuous PDF of epistemic uncertainty $x$ is the curve of the lower bound at different points $x^{\tau}$.

Step 8: The continuous PDF of epistemic uncertainty $x$ is applied to the uncertainty propagation and reliability-based optimization design of engineering products.

\section{Numerical and Engineering Examples}

Two numerical examples and one engineering example are demonstrated to explain the effectiveness of the proposed probabilistic representation approaches for multiple types of epistemic uncertainties. The three examples were calculated using Matlab ${ }^{\circledR} \mathrm{R} 2017 \mathrm{~b}$ on a computer with Inter i5-7400 CPU and 8GB RAM. Numerical example 1 illustrates that the proposed methodology can deal with multiple different types of epistemic uncertainties. In numerical example 2, the proposed methodology is applied to uncertainty propagation analysis for multiple types of epistemic uncertainties, and the probabilistic uncertainty result is similar to the results of the Monte Carlo simulation (MCS) method, while the computational efficiency is improved. In the engineering example, the proposed method can determinate accurate uncertainty bounds of the total heat transfer rate with high computational efficiency.

\subsection{Numerical Example 1}

Five different conditions of the epistemic uncertainty variable $x \in[10,20]$ were analyzed as follows:

Case 1: $x$ is represented using a single interval $[10,20]$;

Case 2: $x$ is represented using multiple intervals $[10,13],[14,16]$, and $[19,20]$;

Case 3: $x$ is represented using a triangular fuzzy number $[10,13,20]$;

Case 4: $x$ is represented using evidence theory, the non-overlapping subintervals are $[10,14]$, $[14,17]$, and $[17,20]$, and the corresponding BPAs are $0.3,0.5$, and 0.2 , respectively; and

Case 5: $x$ is represented using evidence theory, the overlapping subintervals are $[10,15],[13,18]$, and $[17,20]$, and the corresponding BPAs are $0.3,0.5$, and 0.2 , respectively.

The uncertain design intervals $\left[x_{\text {lower }}, x_{\text {upper }}\right]$ of epistemic uncertainty are the same $[10,20]$ in Case 1-Case 5 according to the algorithm presented in Section 4.2. The bounds of four statistical moments $M_{1} \sim M_{4}$ of $x$ in Case 1-Case 5 were calculated according to the methods presented in Section 3, and the results are listed in Table 2. The converge procedure used to calculate the bounds of $M_{2}$ in Case 2 is shown in Figure 5. A series of sampling points of $M_{1} \sim M_{4}$ were randomly selected and the corresponding cubic normal distribution functions were constructed. In total, 101 sampling points $x^{\tau}=10+0.1(\tau-1)(\tau=1, \cdots, 101)$ were selected by dividing the uncertain design interval $[10,20]$ uniformly. The CDFs at these sampling points $x^{\tau}$ with different $M_{1} \sim M_{4}$ values in Case 1-Case 5 were calculated and are shown in Figure 6. The maximum and minimum CDF values at $x^{\tau}$ were calculated by comparing the CDF under every $x^{\tau}$, and the probabilistic representation functions of $x$ in Case 1-Case 5 are shown in Figure 7. Results indicate that the epistemic uncertainty with different representation functions can be probabilistically represented using the proposed algorithm. Compared with the methodology presented in [35], the proposed algorithm extends the application of the cubic 
normal transformation method from interval variables to multiple types of epistemic uncertainties. The uncertain interval can be decreased by obtaining more uncertainty information. Only a single interval was available in Case 1, and the uncertainty interval at every value of $x$ was large, while the evidence theory and BPAs of three subintervals were available in Case 4 and Case 5, and the uncertainty interval was smaller than that in Case 1.



Figure 5. Optimization iteration procedure of calculating bounds of $M_{2}$ in Case 2.

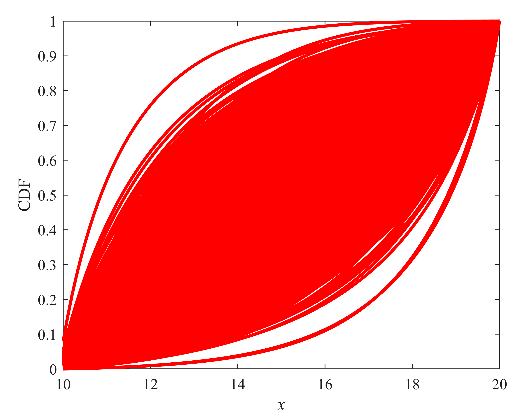

(a)

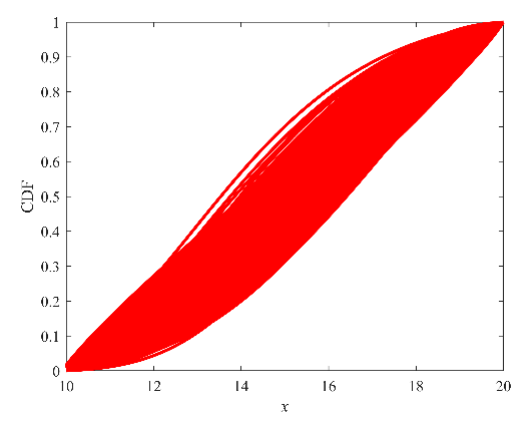

(b)

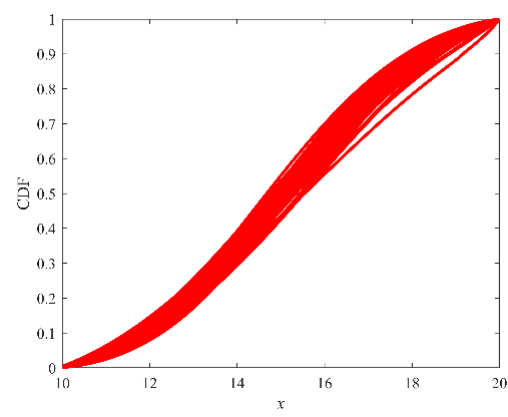

(d)

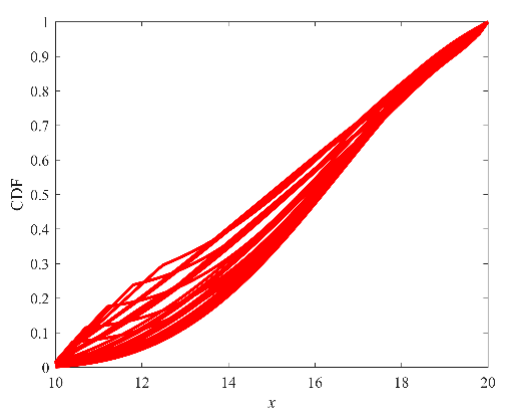

(c)

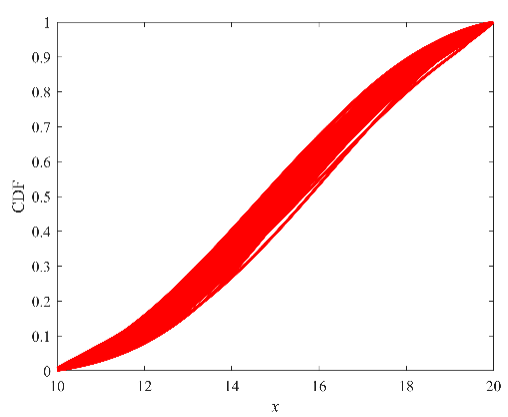

(e)

Figure 6. Cumulative probability density function of epistemic uncertainties (a) Case 1; (b) Case 2; (c) Case 3; (d) Case 4; (e) Case 5. 


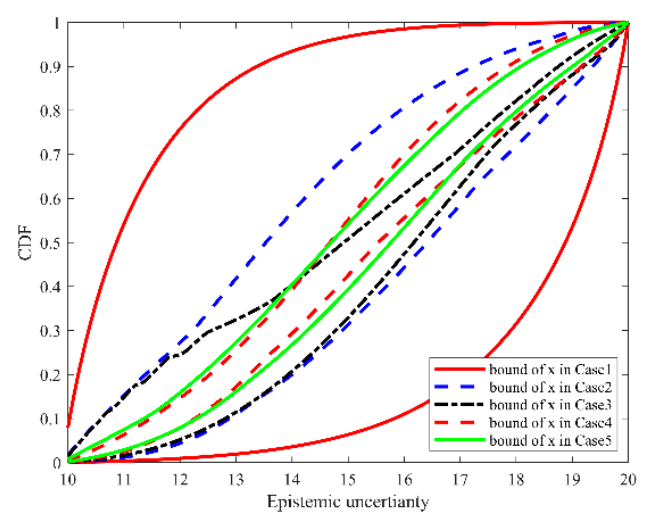

Figure 7. Cumulative probability density function comparison of epistemic uncertainties with different types of uncertainty information.

Table 2. Four statistical moments of epistemic uncertainties in example 1.

\begin{tabular}{cccccc}
\hline $\boldsymbol{l}$-th Order & Case 1 & Case 2 & Case 3 & Case 4 & Case 5 \\
\hline$l=1$ & {$[10,20]$} & {$[14.33,16.33]$} & {$[11.50,16.50]$} & {$[14.51,15.59]$} & {$[14.35,15.98]$} \\
$l=2$ & {$[0,25]$} & {$[6.48,16.53]$} & {$[0.75,8.78]$} & {$[4.03,9.04]$} & {$[3.83,9.25]$} \\
$l=3$ & {$[-96.70,96.10]$} & {$[-18.22,19.28]$} & {$[-4.82,21.19]$} & {$[-11.94,6.88]$} & {$[-17.75,10.22]$} \\
$l=4$ & {$[0,835.87]$} & {$[63.06,409.98]$} & {$[1.01,156.05]$} & {$[43.29,161.35]$} & {$[33.60,158.52]$} \\
\hline
\end{tabular}

\subsection{Numerical Example 2}

The mathematical example presented in Equation (20) was used to demonstrate the effectiveness of the proposed algorithm in the uncertainty propagation analysis problem:

$$
y=x_{1}+x_{2}+x_{3}
$$

where $x_{1} \in[2,5]$ is a single interval variable, $x_{2}$ is a triangular fuzzy variable that is represented within $[6,10,11]$, and $x_{3}$ is represented using evidence theory, where the subintervals are $[10,15],[13,18]$, and $[17,20]$, and the corresponding BPAs are $0.3,0.5$, and 0.2 , respectively.

The bounds of four statistical moments $M_{1} \sim M_{4}$ for $x_{1} \sim x_{3}$ were calculated and are listed in Table 3. The probabilistic CDF bounds of $x_{1} \sim x_{3}$ were calculated using the proposed algorithm in Section 4.2, and the results are shown in Figure 8.

The performance function $y$ in Equation (20) monotonically increases according to the increases of $x_{1}, x_{2}$, and $x_{3}$, respectively. Therefore, the upper bound of the CDF of $y$ was calculated directly using the probabilistic uncertainty propagation analysis method with the upper bound of the CDFs of $x_{1}, x_{2}$, and $x_{3}$. The lower bound of the CDF of $y$ was calculated using the probabilistic uncertainty propagation analysis method with the lower bounds of the CDFs of $x_{1}, x_{2}$, and $x_{3}$. The response CDF bounds of $y$ calculated using the proposed unified probabilistic representation approach are shown by dashed lines in Figure 9.

Table 3. Four statistical moments of epistemic uncertainties in example 2.

\begin{tabular}{cccc}
\hline$l$-th Order & $x_{1}$ & $x_{2}$ & $x_{3}$ \\
\hline$l=1$ & {$[2,5]$} & {$[10.50,12.50]$} & {$[14.35,15.98]$} \\
$l=2$ & {$[0,2.25]$} & {$[2.08,6.75]$} & {$[3.83,9.25]$} \\
$l=3$ & {$[-2.60,2.60]$} & {$[-7.14,0]$} & {$[-17.75,10.22]$} \\
$l=4$ & {$[0,6.76]$} & {$[7.81,82.01]$} & {$[33.60,158.52]$} \\
\hline
\end{tabular}




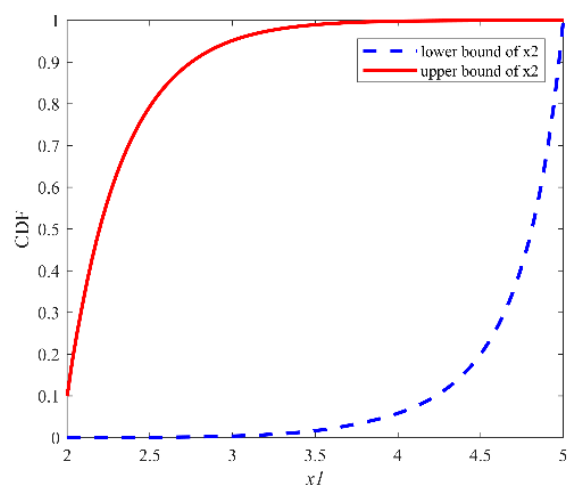

(a)

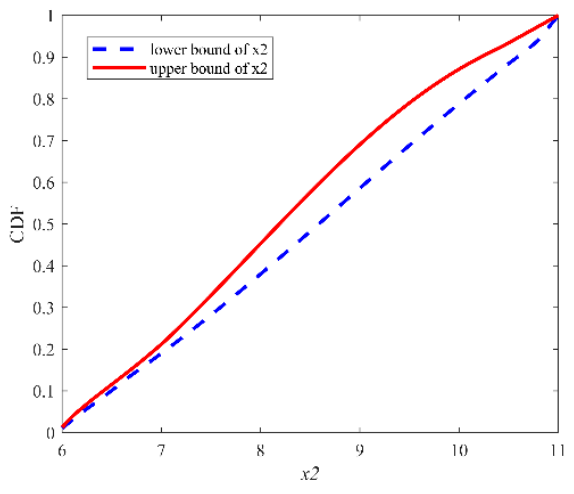

(b)

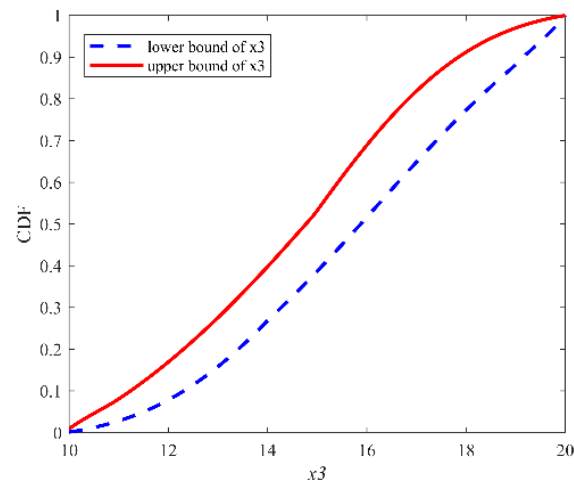

(c)

Figure 8. (a) Probabilistic representation of epistemic uncertain variables $x_{1}$; (b) probabilistic representation of epistemic uncertain variables $x_{2} ;$ (c) probabilistic representation of epistemic uncertain variables $x_{3}$.

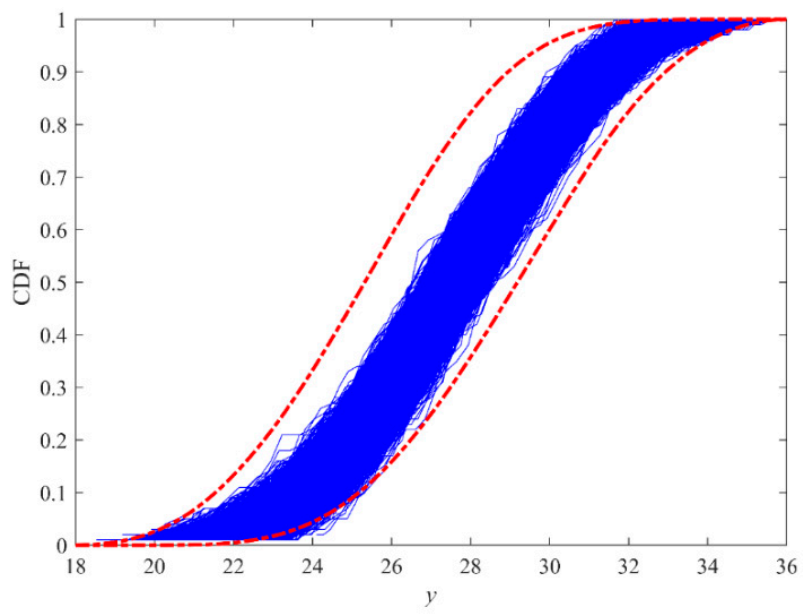

Figure 9. Cumulative probability density function of response $y$.

To verify the effectiveness of the proposed algorithm, the MCS method was also used to solve the uncertainty propagation problem. The results are shown by a series of solid lines in Figure 9 . It can be observed that the CDF bounds calculated using the proposed algorithm cover the CDFs calculated with the MCS methods, which demonstrates the effectiveness of the proposed algorithm. The evaluation number of the calculating performance function $y$ using the MCS method was $10^{5}$, 
while the evaluation number of the proposed algorithm was 202, which shows an improvement in the calculation effectiveness. The time taken to evaluate the calculation of the uncertainty of the performance function using the proposed algorithm and MCS method was 4.75 and $14.67 \mathrm{~s}$, respectively, and the computational efficiency was improved by $67.6 \%$.

\subsection{Engineering Application: Heat Exchanger}

A 24-stream plate-fin heat exchanger was employed to demonstrate the effectiveness of the proposed algorithm in engineering products. The plate-fin heat exchangers have a relatively high heat transfer surface area to volume ratio and have been widely applied in various fields, including air separation equipment, the aerospace industry, and the automobile industry.

The 24-stream heat exchanger was composed of 6 streams of A, B, C, and I, respectively. The deterministic parameters of these streams are listed in Table 4, which are the same as those presented in $[50,51]$. The total heat transfer rate $Q$ is the key index used to evaluate the thermal performance of the heat exchanger, which can be calculated using the integral-mean temperature method (IMTD) method under determinate conditions. However, there are some uncertainties that have deteriorative influences on $Q$. The fin height of stream A $f_{h, A}$ was assumed to be a single interval variable $[0.017 \mathrm{~m}, 0.021 \mathrm{~m}]$. The inlet mass flow rate of stream $\mathrm{A} \dot{m}_{A}$ was a triangular fuzzy number, and the lower bound, upper bound, and most likely value of $\dot{m}_{A}$ were $0.701,0.720$, and $0.716 \mathrm{~kg} / \mathrm{s}$, respectively. The fin space of stream $\mathrm{A} f_{f, A}$ was represented using evidence theory. There were three subintervals $\left[116 \mathrm{~m}^{-1}, 118 \mathrm{~m}^{-1}\right],\left[117 \mathrm{~m}^{-1}, 119 \mathrm{~m}^{-1}\right]$, and $\left[119 \mathrm{~m}^{-1}, 120 \mathrm{~m}^{-1}\right]$, whose BPAs were 0.3 , 0.6 , and 0.1 , respectively. Other parameters were deterministic parameters and are listed in Table 4, which contains the flow direction $j$, inlet temperature $T$, heat transfer coefficient $h$, and specific heat at constant pressure $C_{p}$.

Table 4. Determinate variables of the heat exchanger.

\begin{tabular}{ccccccccc}
\hline Stream & $\begin{array}{c}\text { Direction } \\
\text { of Entry }\end{array}$ & $\begin{array}{c}\mathbf{T} \\
\mathbf{K}\end{array}$ & $\begin{array}{c}\mathbf{h} \\
\mathbf{K w} / \mathbf{m}^{\mathbf{2}} \mathbf{K}\end{array}$ & $\begin{array}{c}\mathbf{C}_{\mathbf{p}} \\
\mathbf{k J} / \mathbf{k g ~ K}\end{array}$ & $\begin{array}{c}\dot{\mathbf{m}} \\
\mathbf{k g} / \mathbf{s}\end{array}$ & $\begin{array}{c}\mathbf{f}_{\mathbf{t}} \\
\mathbf{m}\end{array}$ & $\begin{array}{c}\mathbf{f}_{\mathbf{f}} \\
\mathbf{m}^{-\mathbf{1}}\end{array}$ & $\begin{array}{c}\mathbf{f}_{\mathbf{h}} \\
\mathbf{m}\end{array}$ \\
\hline $\mathrm{A}$ & -1 & 180 & 0.163 & 1.043 & & & 118.50 & \\
$\mathrm{~B}$ & -1 & 170 & 0.094 & 0.915 & 0.191 & $0.254 \times 10^{-3}$ & 244.10 & 0.010 \\
$\mathrm{C}$ & 1 & 300 & 0.116 & 1.006 & 0.455 & $0.813 \times 10^{-3}$ & 78.74 & 0.019 \\
$\mathrm{I}$ & 1 & 320 & 0.116 & 1.076 & 0.305 & $0.813 \times 10^{-3}$ & 78.74 & 0.019 \\
\hline
\end{tabular}

The failure model $G$ is defined in Equation (21):

$$
G=Q\left(f_{h, A}, \dot{m}_{A}, f_{t, A}\right) \geq Q_{\min } .
$$

The total heat transfer rate $Q$ with determinate parameters was calculated using the IMTD method in our previous article [50], where $Q_{\min }$ was the allowable minimum heat transfer rate. The uncertain variables $f_{h, A}, \dot{m}_{A}$, and $f_{f, A}$ were probabilistically represented using the proposed algorithm. The belief and plausibility measures of $Q$ under different $Q_{\min }$ values were calculated using the proposed method and MCS method, respectively, as shown in Figure 10. The bounds calculated using the proposed algorithm are shown by dash lines, while the results of MCS are shown using a series of solid lines. It can be observed that the uncertainty analysis results of the MCS method were enveloped by the proposed algorithm. However, a large number of thermal calculations for the heat exchanger are needed for the MCS method, while only a small functional evaluation is required in the proposed algorithm. The computational time for the proposed methodology and MCS method was 95.3 and $667.2 \mathrm{~s}$, respectively, so the computational efficiency was improved by $85.7 \%$. If the total heat transfer rate $Q$ is calculated using finite element analysis method or long-term experimental test, the evaluation time of calculating $Q$ will be increased compared with the IMTD thermal model, and the computational effectiveness can be further improved by decreasing the evaluation number of 
the calculations of the performance function in the uncertainty analysis. The proposed method can transform multiple epistemic uncertainties into probabilistic uncertain variables with less computational burden. Some probabilistic reliability methodologies can be used to deal with epistemic uncertainties, and the computational complexity and computational efficiency can be improved compared with non-probabilistic methodologies.

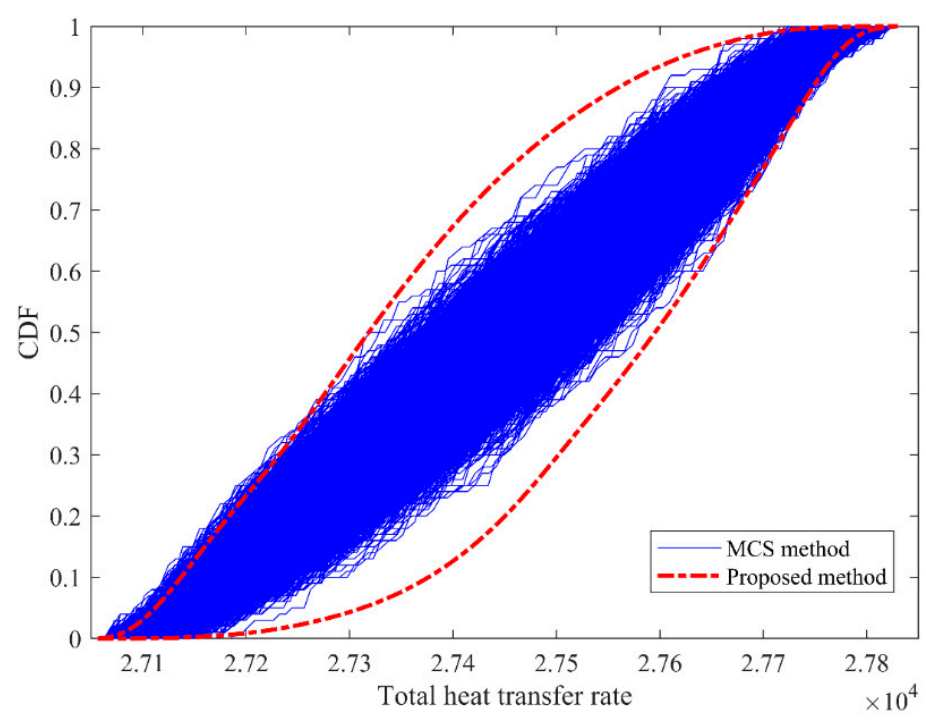

Figure 10. Uncertainty analysis results of the heat exchanger.

\section{Conclusions and Future Works}

This paper extended the application of the cubic normal transformation method from interval variables to multiple types of epistemic uncertainties and developed a unified probabilistic representation framework for uncertainty propagation analysis of multiple types of epistemic uncertainties. A unified probabilistic representation approach was proposed. The uncertain intervals of four statistical moments were calculated for epistemic uncertainties represented by using the interval approach, triangular fuzzy approach, and evidence theory. A construction algorithm of continuous cubic normal distribution function sets was developed based on the uncertain intervals of four statistical moments, and these epistemic uncertainties were represented using continuous probabilistic belief and plausibility measure functions. Compared with the traditional MCS method, the proposed methodology can acquire accurate uncertainty bounds of performance functions with higher computational efficiency. Especially, the computational efficiency in the uncertainty analysis of the total heat transfer rate of the 24-stream plate-fin heat exchanger was improved by $85.7 \%$.

Although some improvements were made for the uncertainty propagation analysis of multiple types of epistemic uncertainty, the proposed algorithms have some limitations, which can be further researched in the future:

(1). The uncertainty representation results could be integrated into probabilistic reliability optimization methodologies. The lower and upper bounds of the probability density function of the performance function could be applied into first-order or second-order reliability methods. Reliability optimization algorithms could be developed to solve the reliability problem considering multiple types of epistemic uncertainties.

(2). The proposed uncertainty representation method could be integrated with an adaptive approximation method to solve the reliability problem of complex engineering products. Though the proposed algorithm can decrease the evaluation number required to calculate the performance function in the uncertainty propagation analysis, the performance function needs to be calculated several times in the reliability optimization process. Therefore, determining how to construct an adaptive 
approximation model for probabilistic reliability optimization under multiple types of epistemic uncertainties is an issue worth researching in the future.

(3). The probabilistic uncertainty representation framework can be extended to integrate more epistemic uncertainty types, such as $p$-box, convex model, insufficient data, and variables with uncertainty distribution parameters.

Author Contributions: Conceptualization, X.P.; Methodology, X.P. and Q.G.; Supervision, Z.L. and S.J.; Writing — original draft, X.P. and Q.G.; Writing — review and editing, J.L. and B.Y. All authors have read and agreed to the published version of the manuscript.

Funding: This research was funded by the National Key Research and Development Program of China [under grant number 2017YFB0603704], the National Natural Science Foundation of China under grant [number: 51875525, U1610112, U1608256], the Zhejiang Provincial Natural Science Foundation of China under grant [number: LY20E050020], and Open Foundation of the State Key Laboratory of Fluid Power and Mechatronic Systems [number: GZKF-201916].

Conflicts of Interest: No potential conflict of interest was reported by the authors.

\section{References}

1. Keshtegar, B.; Meng, Z. A hybrid relaxed first-order reliability method for efficient structural reliability analysis. Struct. Saf. 2017, 66, 84-93. [CrossRef]

2. Cheng, J.; Tang, M.; Liu, Z.; Tan, J. Direct reliability-based design optimization of uncertain structures with interval parameters. J. Zhejiang Univ. Sci. A 2016, 17, 841-854. [CrossRef]

3. Beck, A.T.; Tessari, R.K.; Kroetz, H.M. System reliability-based design optimization and risk-based optimization: A benchmark example considering progressive collapse. Eng. Optim. 2019, 51, 1000-1012. [CrossRef]

4. Strömberg, N. Reliability-based design optimization using SORM and SQP. Struct. Multidiscip. Optim. 2017, 56, 631-645. [CrossRef]

5. Hu, Z.; Du, X. Efficient reliability-based design with second order approximations. Eng. Optim. 2019, 51, 101-119. [CrossRef]

6. Meng, Z.; Yang, D.; Zhou, H.; Yu, B. An accurate and efficient reliability-based design optimization using the second order reliability method and improved stability transformation method. Eng. Optim. 2018, 50, 749-765. [CrossRef]

7. $\mathrm{Hu}, \mathrm{Z}$.; Mahadevan, S.; Du, X. Uncertainty quantification of time-dependent reliability analysis in the presence of parametric uncertainty. ASCE-ASME J. Risk Uncert. Eng. Syst. Part B Mech. Eng. 2016, 2, 031005. [CrossRef]

8. das Neves Carneiro, G.; Antonio, C.C. A RBRDO approach based on structural robustness and imposed reliability level. Struct. Multidiscip. Optim. 2018, 57, 2411-2429. [CrossRef]

9. Oberkampf, W.L.; Helton, J.C.; Joslyn, C.A.; Wojtkiewicz, S.F.; Ferson, S. Challenge problems: Uncertainty in system response given uncertain parameters. Reliab. Eng. Syst. Saf. 2004, 85, 11-19. [CrossRef]

10. Der Kiureghian, A.; Ditlevsen, O. Aleatory or epistemic? Does it matter? Struct. Saf. 2009, 31, 105-112. [CrossRef]

11. Peng, X.; Guo, Y.; Qiu, C.; Wu, H.; Li, J.; Chen, G.; Jiang, S.; Liu, Z. Reliability optimization design for composite laminated plate considering multiple types of uncertain parameters. Eng. Optim. 2020, 52, 1-16. [CrossRef]

12. Beer, M.; Ferson, S.; Kreinovich, V. Imprecise probabilities in engineering analyses. Mech. Syst. Signal Process. 2013, 37, 4-29. [CrossRef]

13. Cheng, J.; Liu, Z.; Tang, M.; Tan, J. Robust optimization of uncertain structures based on normalized violation degree of interval constraint. Comput. Struct. 2017, 182, 41-54. [CrossRef]

14. Hurtado, J.E.; Alvarez, D.A.; Paredes, J.A. Interval reliability analysis under the specification of statistical information on the input variables. Struct. Saf. 2017, 65, 35-48. [CrossRef]

15. Jiang, C.; Han, X.; Lu, G.Y.; Liu, J.; Zhang, Z.; Bai, Y.C. Correlation analysis of non-probabilistic convex model and corresponding structural reliability technique. Comput. Methods Appl. Mech. Eng. 2011, 200, 2528-2546. [CrossRef] 
16. Meng, Z.; Hu, H.; Zhou, H. Super parametric convex model and its application for non-probabilistic reliability-based design optimization. Appl. Math. Model. 2018, 55, 354-370. [CrossRef]

17. Dey, S.; Mukhopadhyay, T.; Khodaparast, H.H.; Adhikari, S. Fuzzy uncertainty propagation in composites using Gram-Schmidt polynomial chaos expansion. Appl. Math. Model 2016, 40, 4412-4428. [CrossRef]

18. Huang, Z.; Jiang, W.; Tang, Y. A new method to evaluate risk in failure mode and effects analysis under fuzzy information. Soft Comput. 2018, 22, 4779-4787. [CrossRef]

19. Bae, H.-R.; Grandhi, R.V.; Canfield, R.A. Epistemic uncertainty quantification techniques including evidence theory for large-scale structures. Comput. Struct. 2004, 82, 1101-1112. [CrossRef]

20. Zhang, Z.; Jiang, C.; Wang, G.G.; Han, X. First and second order approximate reliability analysis methods using evidence theory. Reliab. Eng. Syst. Saf. 2015, 137, 40-49. [CrossRef]

21. Yang, X.; Liu, Y.; Ma, P. Structural reliability analysis under evidence theory using the active learning kriging model. Eng. Optim. 2017, 49, 1922-1938. [CrossRef]

22. Huang, Z.; Xu, J.; Yang, T.; Li, F.; Deng, S. Evidence-Theory-Based Robust Optimization and Its Application in Micro-Electromechanical Systems. Appl. Sci. 2019, 9, 1457. [CrossRef]

23. Ni, B.Y.; Jiang, C.; Huang, Z.L. Discussions on non-probabilistic convex modelling for uncertain problems. Appl. Math. Model. 2018, 59, 54-85. [CrossRef]

24. Suryawanshi, A.; Ghosh, D. Reliability based optimization in aeroelastic stability problems using polynomial chaos based metamodels. Struct. Multidiscip. Optim. 2015, 53, 1069-1080. [CrossRef]

25. Nannapaneni, S.; Mahadevan, S. Reliability analysis under epistemic uncertainty. Reliab. Eng. Syst. Saf. 2016, 155, 9-20. [CrossRef]

26. Bae, H.-R.; Grandhi, R.V.; Canfield, R.A. An approximation approach for uncertainty quantification using evidence theory. Reliab. Eng. Syst. Saf. 2004, 86, 215-225. [CrossRef]

27. Jiang, C.; Zheng, J.; Han, X. Probability-interval hybrid uncertainty analysis for structures with both aleatory and epistemic uncertainties: A review. Struct. Multidiscip. Optim. 2017, 57, 2485-2502. [CrossRef]

28. Peng, X.; Ye, T.; Li, J.; Wu, H.; Jiang, S.; Chen, G. Multi-scale uncertainty quantification of composite laminated plate considering random and interval variables with data driven PCE method. Mech. Adva. Mater. Struct. 2020, 27. [CrossRef]

29. Cheng, J.; Zhang, Y.; Feng, Y.; Liu, Z.; Tan, J. Structural Optim. of a High-Speed Press Considering Multi-Source Uncertainties Based on a New Heterogeneous TOPSIS. Appl. Sci. 2018, 8, 126. [CrossRef]

30. Sankararaman, S.; Mahadevan, S. Distribution type uncertainty due to sparse and imprecise data. Mech. Syst. Signal Process. 2013, 37, 182-198. [CrossRef]

31. Sankararaman, S.; Mahadevan, S. Likelihood-based representation of epistemic uncertainty due to sparse point data and/or interval data. Reliab. Eng. Syst. Saf. 2011, 96, 814-824. [CrossRef]

32. Sankararaman, S.; Mahadevan, S. Separating the contributions of variability and parameter uncertainty in probability distributions. Reliab. Eng. Syst. Saf. 2013, 112, 187-199. [CrossRef]

33. Zaman, K.; McDonald, M.; Mahadevan, S. Probabilistic framework for uncertainty propagation with both probabilistic and interval variables. J. Mech. Des. 2011, 133, 021010. [CrossRef]

34. Zaman, K.; Dey, P.R. Likelihood-based representation of epistemic uncertainty and its application in robustness-based design optimization. Struct. Multidiscip. Optim. 2017, 56, 767-780. [CrossRef]

35. Zaman, K.; Rangavajhala, S.; McDonald, M.P.; Mahadevan, S. A probabilistic approach for representation of interval uncertainty. Reliab. Eng. Syst. Saf. 2011, 96, 117-130. [CrossRef]

36. Cho, H.; Choi, K.K.; Gaul, N.J.; Lee, I.; Lamb, D.; Gorsich, D. Conservative reliability-based design optimization method with insufficient input data. Struct. Multidiscip. Optim. 2016, 54, 1609-1630. [CrossRef]

37. Lü, H.; Shangguan, W.-B.; Yu, D. A unified method and its application to brake instability analysis involving different types of epistemic uncertainties. Appl. Math. Model. 2018, 56, 158-171. [CrossRef]

38. Zhang, J.; Shields, M.D. On the quantification and efficient propagation of imprecise probabilities resulting from small datasets. Mech. Syst. Signal Process. 2018, 98, 465-483. [CrossRef]

39. Zhao, Y.-G.; Zhang, X.-Y.; Lu, Z.-H. Complete monotonic expression of the fourth-moment normal transformation for structural reliability. Comput. Struct. 2018, 196, 186-199. [CrossRef]

40. Zhao, Y.-G.; Zhang, X.-Y.; Lu, Z.-H. A flexible distribution and its application in reliability engineering. Reliab. Eng. Syst. Saf. 2018, 176, 1-12. [CrossRef]

41. Zhang, Z.; Ruan, X.X.; Duan, M.F.; Jiang, C. An efficient epistemic uncertainty analysis method using evidence theory. Comput. Methods Appl. Mech. Eng. 2018, 339, 443-466. [CrossRef] 
42. Liu, H.B.; Jiang, C.; Jia, X.Y.; Long, X.Y.; Zhang, Z.; Guan, F.J. A new uncertainty propagation method for problems with parameterized probability-boxes. Reliab. Eng. Syst. Saf. 2018, 172, 64-73. [CrossRef]

43. Dey, A.; Zaman, K. A robust optimization approach for solving two-person games under interval uncertainty. Comput. Oper. Res. 2020, 119, 104937. [CrossRef]

44. Dang, C.; Xu, J. Novel algorithm for reconstruction of a distribution by fitting its first-four statistical moments. Appl. Math. Model. 2019, 71,505-524. [CrossRef]

45. Zhang, L.-W. An improved fourth-order moment reliability method for strongly skewed distributions. Struct. Multidiscip. Optim. 2020, 61, 1-13. [CrossRef]

46. Lu, H.; Cao, S.; Zhu, Z.; Zhang, Y. An improved high order moment-based saddlepoint approximation method for reliability analysis. Appl. Math. Model. 2020, 82, 836-847. [CrossRef]

47. Zadeh, L.A. Fuzzy sets. Inf. Control 1965, 8, 338-353. [CrossRef]

48. Zaman, K.; McDonald, M.; Mahadevan, S.; Green, L. Robustness-based design optimization under data uncertainty. Struct. Multidiscip. Optim. 2011, 44, 183-197. [CrossRef]

49. Liou, T.-S.; Wang, M.-J.J. Ranking fuzzy numbers with integral value. Fuzzy Sets Syst. 1992, 50, $247-255$. [CrossRef]

50. Peng, X.; Liu, Z.; Qiu, C.; Tan, J. Passage arrangement design for multi-stream plate-fin heat exchanger under multiple operating conditions. Int. J. Heat Mass Transf. 2014, 77, 1055-1062. [CrossRef]

51. Ghosh, S.; Ghosh, I.; Pratihar, D.K.; Maiti, B.; Das, P.K. Optimum stacking pattern for multi-stream plate-fin heat exchanger through a genetic algorithm. Int J. Therm. Sci. 2011, 50, 214-224. [CrossRef]

(C) 2020 by the authors. Licensee MDPI, Basel, Switzerland. This article is an open access article distributed under the terms and conditions of the Creative Commons Attribution (CC BY) license (http://creativecommons.org/licenses/by/4.0/). 